\title{
Analytic solutions of transcendental equations with application to automatics
}

\author{
HENRYK GÓRECKI and MIECZYSŁAW ZACZYK
}

In the paper the extremal dynamic error $x(\tau)$ and the moment of time $\tau$ are considered. The extremal value of dynamic error gives information about accuracy of the system. The time $\tau$ gives information about velocity of transient. The analytical formulae enable design of the system with prescribed properties. These formulae are calculated due to the assumption that $x(\tau)$ is a function of the roots $s_{1}, \ldots, s_{n}$ of the characteristic equation.

Key words: extremal problems, characteristic equation, transmittance.

\section{Introduction}

In the paper [1] the necessary conditions for the extremal value $x(\tau)$ are presented. In the article [2] the method of the decomposition of $n$th order system into a set of 2-nd order systems is given. In this article some new results are obtained.

\section{Statement of the problem}

Calculation of conditions and extremum of the extreme value of the dynamic error [1]. Let us consider the differential equation determining the dynamic error in a linear control system of $n$th order with lumped and constant parameters:

$$
\frac{d^{n} x}{d t^{n}}+a_{1} \frac{d^{n-1} x}{d t^{n-1}}+\cdots+a_{n-1} \frac{d x}{d t}+a_{n} x=0 .
$$

The initial conditions are determined by the force function and the system's parameters. Let us assume in general, that

$$
x^{(i)}(0)=c_{i+1} \neq 0 \text { for } i=0,1, \ldots, n-1
$$

The authors are with AGH University of Science and Technology, Department of Automatics and Biomedical Engineering, Al. Mickiewicza 30, 30-059 Krakow, Poland. E-mails: head@agh.edu.pl, zaczyk@agh.edu.pl

Received 10.05.2016. Revised 6.11.2016. 
We assume further that the characteristic equation of equation (1) has $m$ different real roots and $2 p$ different complex roots.

It is evident that

$$
m+2 p=n .
$$

We denote by $s_{k}$ real roots and

$$
\alpha_{k}+j \omega_{k}=r_{k}, \quad \alpha_{k}-j \omega_{k}=\hat{r}_{k}(k=1,2, \ldots, p) .
$$

The solution of equation (1) takes the form

$$
x(t)=\sum_{k=1}^{m} A_{k} e^{s_{k} t}+\sum_{k=1}^{p}\left[B_{k} \cos \left(\omega_{k} t\right)+C_{k} \sin \left(\omega_{k} t\right)\right] e^{\alpha_{k} t}
$$

where $A_{k}, B_{k}, C_{k}, s_{k}, \alpha_{k}$ and $\omega_{k}$ are real numbers.

The necessary conditions for the dynamic error $x(t)$ to attain an extreme value at $t=\tau$ is given by the relation:

$$
\begin{aligned}
& \frac{d x}{d t}=\sum_{k=1}^{m} A_{k} s_{k} e^{s_{k} \tau}+ \\
& +\sum_{k=1}^{p}\left[\left(-B_{k} \sin \omega_{k} \tau+C_{k} \cos \omega_{k} \tau\right) \omega_{k}+\left(B_{k} \cos \omega_{k} \tau+C_{k} \sin \omega_{k} \tau\right) \alpha_{k}\right] e^{\alpha_{k} \tau}=0 .
\end{aligned}
$$

The constants are determined from

$$
x^{(i)}(0)=c_{i+1}=\sum_{k=1}^{m} A_{k} s_{k}^{i}+\sum_{k=1}^{p}\left[B_{k} \operatorname{Re}\left(r_{k}^{i}\right)+C_{k} \operatorname{Im}\left(r_{k}^{i}\right)\right](i=0,1, \ldots, n-1) .
$$

The extreme value of the dynamic error is

$$
x(\tau)=\sum_{k=1}^{m} A_{k} e^{s_{k} \tau}+\sum_{k=1}^{p}\left[B_{k} \cos \left(\omega_{k} \tau\right)+C_{k} \sin \left(\omega_{k} \tau\right)\right] e^{\alpha_{k} \tau} .
$$

The extremum of extreme value of the dynamic error given by equation (5), computed with regard to the parameters $s_{k}, \alpha_{k}, \omega_{k}$, is obtained by putting the respective partial derivatives of $x(\tau)$ equal to zero.

Denoting by

$$
\left(\frac{\partial x(\tau)}{\partial s_{k}}\right)^{*},\left(\frac{\partial x(\tau)}{\partial \alpha_{k}}\right)^{*},\left(\frac{\partial x(\tau)}{\partial \omega_{k}}\right)^{*}
$$


the partial derivatives of expression (5) for constant $\tau$ we may write

$$
\left.\begin{array}{rl}
\frac{\partial x(\tau)}{\partial s_{k}} & =\left(\frac{\partial x(\tau)}{\partial s_{k}}\right)^{*}+\frac{\partial x(\tau)}{\partial \tau} \frac{\partial \tau}{\partial s_{k}} \\
\frac{\partial x(\tau)}{\partial \alpha_{k}} & =\left(\frac{\partial x(\tau)}{\partial \alpha_{k}}\right)^{*}+\frac{\partial x(\tau)}{\partial \tau} \frac{\partial \tau}{\partial \alpha_{k}} \\
\frac{\partial x(\tau)}{\partial \omega_{k}} & =\left(\frac{\partial x(\tau)}{\partial \omega_{k}}\right)^{*}+\frac{\partial x(\tau)}{\partial \tau} \frac{\partial \tau}{\partial \omega_{k}}
\end{array}\right\} .
$$

However, we have from equation (3)

$$
\frac{\partial x(\tau)}{\partial \tau}=0
$$

and therefore

$$
\left.\begin{array}{rl}
\frac{\partial x(\tau)}{\partial s_{k}} & =\left(\frac{\partial x(\tau)}{\partial s_{k}}\right)^{*} \\
\frac{\partial x(\tau)}{\partial \alpha_{k}} & =\left(\frac{\partial x(\tau)}{\partial \alpha_{k}}\right)^{*} \\
\frac{\partial x(\tau)}{\partial \omega_{k}} & =\left(\frac{\partial x(\tau)}{\partial \omega_{k}}\right)^{*}
\end{array}\right\} .
$$

We obtain the following conditions:

$$
\left.\begin{array}{l}
\sum_{k=1}^{m} \frac{\partial A_{k}}{\partial s_{j}} e^{s_{k} \tau}+A_{j} \tau e^{s_{j} \tau}+\sum_{k=1}^{p}\left(\frac{\partial B_{k}}{\partial s_{j}} \cos \omega_{k} \tau+\frac{\partial C_{k}}{\partial s_{j}} \sin \omega_{k} \tau\right) e^{\alpha_{k} \tau}=0 \\
\sum_{k=1}^{m} \frac{\partial A_{k}}{\partial \alpha_{j}} e^{s_{k} \tau}+ \\
+\sum_{k=1}^{p}\left(\frac{\partial B_{k}}{\partial \alpha_{j}} \cos \omega_{k} \tau+\frac{\partial C_{k}}{\partial \alpha_{j}} \sin \omega_{k} \tau\right) e^{\alpha_{k} \tau}+\left(B_{j} \cos \omega_{j} \tau+C_{j} \sin \omega_{j} \tau\right) e^{\alpha_{j} \tau} \tau=0 \\
\sum_{k=1}^{m} \frac{\partial A_{k}}{\partial \omega_{j}} e^{s_{k} \tau}+\quad \\
+\sum_{k=1}^{p}\left(\frac{\partial B_{k}}{\partial \omega_{j}} \cos \omega_{k} \tau+\frac{\partial C_{k}}{\partial \omega_{j}} \sin \omega_{k} \tau\right) e^{\alpha_{k} \tau}+\left(C_{j} \cos \omega_{j} \tau-B_{j} \sin \omega_{j} \tau\right) e^{\alpha_{j} \tau} \tau=0 \\
j=1,2, \ldots, p
\end{array}\right\}
$$

In this way we have a system of $n$ linear and homogenous equations with $n$ unknowns $e^{s_{k} \tau}, e^{\alpha_{k} \tau} \sin \omega_{k} \tau, e^{\alpha_{k} \tau} \cos \omega_{k} \tau$. 
The determinant of system (8) must vanish if there are not to be all zero solutions. The same determinant (after being reflected about one of the main diagonals) is:

$$
|D+A \tau|
$$

where $D$ and $A$ are matrices determined by the following equations:

$$
\begin{gathered}
D=\sum_{j=1}^{m} \sum_{k=1}^{m} \frac{\partial A_{j}}{\partial s_{k}} E_{j k}+\sum_{j=1}^{p} \sum_{k=1}^{m}\left(\frac{\partial B_{j}}{\partial s_{k}} E_{m+2 j-1, k}+\frac{\partial C_{j}}{\partial s_{k}} E_{m+2 j, k}\right)+ \\
+\sum_{j=1}^{m} \sum_{k=1}^{p}\left(\frac{\partial A_{j}}{\partial \alpha_{k}} E_{j, m+2 k-1}+\frac{\partial A_{j}}{\partial \omega_{k}} E_{j, m+2 k}\right)+ \\
+\sum_{j=1}^{p} \sum_{k=1}^{p}\left[\left(\frac{\partial B_{j}}{\partial \alpha_{k}} E_{m+2 j-1, m+2 k-1}+\frac{\partial B_{j}}{\partial \omega_{k}} E_{m+2 j-1, m+2 k}\right)+\right. \\
\left.+\left(\frac{\partial C_{j}}{\partial \alpha_{k}} E_{m+2 j, m+2 k-1}+\frac{\partial C_{j}}{\partial \omega_{k}} E_{m+2 j, m+2 k}\right)\right] \\
A=\sum_{j=1}^{m} A_{j} E_{j j}+\sum_{j=1}^{p}\left[B_{j}\left(E_{m+2 j-1, m+2 j-1}-E_{m+2 j, m+2 j}\right)+\right. \\
\left.+C_{j}\left(E_{m+2 j-1, m+2 j}+E_{m+2 j, m+2 j-1}\right)\right] \\
E_{j k}=\left(e_{\mu, v}^{(j k)}\right) \quad \mu, v=1, \ldots, n \\
e_{\mu, p}^{(j k)}=\delta_{\mu j} \delta_{v k}=\left\{\begin{array}{l}
1 \text { for } \mu=j, v=k \\
0 \text { for all other cases. }
\end{array}\right.
\end{gathered}
$$

Finally we have

$$
|D+A \tau|=0
$$

and system (8) yields for unknown $\tau$ (after some algebraic manipulations) the following equation:

$$
(-1)^{n} \tau^{n} \prod_{k=1}^{m} A_{k} \prod_{k=1}^{p}\left(B_{k}^{2}+C_{k}^{2}\right)=0 .
$$

From relation (13) it is evident that the necessary conditions for $x\left[\tau\left(s_{1}, s_{2}, \ldots, s_{n}\right)\right]$ to have an extremum with respect to $\left(s_{1}, s_{2}, \ldots, s_{n}\right)$ are

$$
\tau=0
$$

which means that

$$
c_{2}=0
$$

or

$$
A_{k}=0 \text { for } k=1,2, \ldots, m
$$


or

$$
\left(B_{k}^{2}+C_{k}^{2}\right)=0 \text { for } k=1,2, \ldots, p .
$$

By applying Laplace transformation to the equation (1) we obtain the transform $X(s)$ of the error $x(t)$.

$$
\begin{aligned}
& X(s)= \\
& \frac{s^{n-1} c_{1}+\left(a_{1} c_{1}+c_{2}\right) s^{n-2}+\left(a_{2} c_{1}+a_{1} c_{2}+c_{3}\right) s^{n-3}+\cdots+\left(a_{n-1} c_{1}+a_{n-2} c_{2}+\ldots+a_{1} c_{n-1}+c_{n}\right)}{s^{n}+a_{1} s^{n-1}+a_{2} s^{n-2}+\cdots+a_{n-1} s+a_{n}} \\
& =\frac{L(s)}{M(s)} .
\end{aligned}
$$

The coefficients $A_{k}, B_{k}, C_{k}$ as we know, are equal

$$
A_{k}=\frac{L\left(s_{k}\right)}{\left[\frac{d M(s)}{d s}\right]_{s=s_{k}}} \quad k=1,2, \ldots, m
$$

and they can attain zero value if the Sylvester's determinant of the polynomials $L(s)$ and $M(s)$ is equal zero. We obtain the following theorem:

Theorem 2 The vanishing of the coefficients $A_{k}(16)$ or $\left(B_{k}^{2}+C_{k}^{2}\right)$ (17) is possible if the numerator $L(s)$ and the denominator $M(s)$ of the transform $X(s)$ have a common root, it means $A_{k}=0$ if $L\left(s_{k}\right)=0$ and $M\left(s_{k}\right)=0$.

In order to eliminate the root $s_{k}$ which will satisfy both equations $L(s)=0$ and $M(s)=0$ we can also use Euclides algorithm [2].

The division of $M(s)$ by $L(s)$ gives

$$
s_{1}=\frac{c_{2}}{c_{1}}
$$

for arbitrary natural power $n=1,2, \ldots$

In the particular cases, which are often in practice, $c_{2}=0$, then from (20) we obtain $s_{1}=0$ [4]. This value is not useful, because this is limit of stability of (1). In this paper these particular cases, using another method, are considered for $c_{2}=0, c_{1} \neq 0, c_{3} \neq 0$ [3].

\section{Solution of the problem}

Let us consider the equation for $n=3$

$$
x(t)=\sum_{i=1}^{3} A_{i} e^{s_{i} t}
$$


with the initial conditions: $x(0)=c_{1}, x^{(1)}(0)=c_{2}, x^{(2)}(0)=c_{3}$. The characteristic equation is

$$
s^{3}+a_{1} s^{2}+a_{2} s+a_{3}=0
$$

where $a_{1}, a_{2}, a_{3}>0$ and $a_{1} a_{2}-a_{3}>0$, are conditions for stability. Let us assume that roots of the equation (22) fulfill the relation

$$
s_{3}=\frac{s_{1}+s_{2}}{2} \text { and } s_{1}<s_{3}<s_{2} .
$$

This assumption denotes that localization of the roots can satisfy one of the three possibilities (Fig. 1). The roots of equation (22) are determined by the relation (23) and Vieta's formulae

$$
a_{1}=-\left(s_{1}+s_{2}+s_{3}\right)=-3 s_{3}
$$

then

$$
s_{3}=-\frac{1}{3} a_{1}
$$
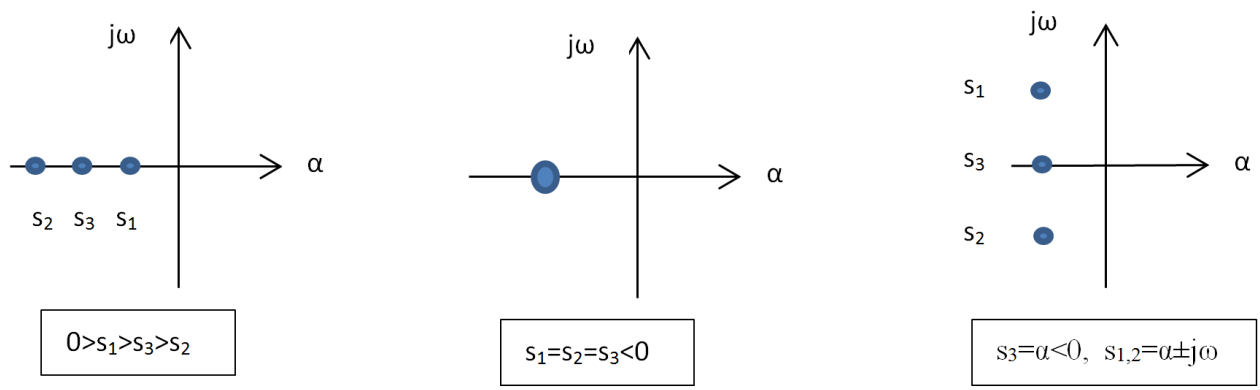

Figure 1: Localization of roots.

The division of the equation (22) by the root $s_{3}$ gives

$$
s^{2}+\frac{2}{3} a_{1} s+a_{2}-\frac{2}{9} a_{1}^{2}=0
$$

and

$$
a_{3}=\frac{1}{3} a_{1} a_{2}-\frac{2}{27} a_{1}^{3}
$$

The roots of equation (25) are

$$
s_{1,2}=-\frac{1}{3} a_{1} \pm \sqrt{\frac{1}{3} a_{1}^{2}-a_{2}} .
$$

From the relation (27) we deduce:

$1^{0}$ If $\frac{1}{3} a_{1}^{2}-a_{2}>0$ there are three real, different roots $s_{1} \neq s_{2} \neq s_{3}$ (24) and (27), 
$2^{0}$ If $\frac{1}{3} a_{1}^{2}-a_{2}=0$ there is one triple root

$$
s_{1}=-\frac{1}{3} a_{1}
$$

$3^{0}$ If $\frac{1}{3} a_{1}^{2}-a_{2}<0$ there is one real root $s_{3}=-\frac{1}{3} a_{1}$,

$$
s_{3}=-\frac{1}{3} a_{1}
$$

and two complex roots

$$
s_{1,2}=\alpha \pm j \omega
$$

where

$$
\left.\begin{array}{c}
\alpha=-\frac{1}{3} a_{1} \\
\omega=\sqrt{a_{2}-\frac{1}{3} a_{1}^{2}}
\end{array}\right\} .
$$

The coefficients of equation (21) are

$$
\left.\begin{array}{l}
A_{1}=\frac{c_{3}-c_{2}\left(s_{2}+s_{3}\right)+c_{1} s_{2} s_{3}}{\left(s_{1}-s_{2}\right)\left(s_{1}-s_{3}\right)} \\
A_{2}=\frac{c_{3}-c_{2}\left(s_{1}+s_{3}\right)+c_{1} s_{1} s_{3}}{\left(s_{1}-s_{2}\right)\left(s_{3}-s_{2}\right)} \\
A_{3}=\frac{c_{3}-c_{2}\left(s_{1}+s_{2}\right)+c_{1} s_{1} s_{2}}{\left(s_{1}-s_{3}\right)\left(s_{2}-s_{3}\right)}
\end{array}\right\} .
$$

From the necessary condition for extremum $x(t)$ determined by (21) we have

$$
\frac{d x}{d t}=s_{1} A_{1} e^{s_{1} \tau}+s_{2} A_{2} e^{s_{2} \tau}+s_{3} A_{3} e^{s_{3} \tau}=0 .
$$

After division of (33) by $e^{s_{3} \tau}$ we obtain

$$
s_{1} A_{1} e^{\left(s_{1}-s_{3}\right) \tau}+s_{2} A_{2} e^{\left(s_{2}-s_{3}\right) \tau}+s_{3} A_{3}=0 .
$$

From the relation (23) we obtain a very important relation

$$
s_{1}-s_{3}=-\left(s_{2}-s_{3}\right)
$$

which substituted to (34) gives

$$
s_{1} A_{1} e^{\left(s_{1}-s_{3}\right) \tau}+s_{2} A_{2} e^{-\left(s_{1}-s_{3}\right) \tau}+s_{3} A_{3}=0 .
$$

After multiplying (36) by $e^{\left(s_{1}-s_{3}\right) \tau}$ we obtain finally

$$
s_{1} A_{1} e^{2\left(s_{1}-s_{3}\right) \tau}+s_{3} A_{3} e^{\left(s_{1}-s_{3}\right) \tau}+s_{2} A_{2}=0 .
$$


It is quadratic equation with respect to $e^{\left(s_{1}-s_{3}\right) \tau}$, then we have:

In the case $1^{0}$, that is of real $s_{1} \neq s_{2} \neq s_{3}$ and $\frac{1}{3} a_{1}^{2}-a_{2}>0$

$$
\tau_{1,2}=\frac{1}{s_{1}-s_{3}} \ln \left(-\frac{1}{2} \frac{s_{3} A_{3}}{s_{1} A_{1}} \pm \sqrt{\left(\frac{1}{2} \frac{s_{3} A_{3}}{s_{1} A_{1}}\right)^{2}-\frac{s_{2} A_{2}}{s_{1} A_{1}}}\right)
$$

or

$$
\tau_{1,2}=\frac{1}{\sqrt{\frac{1}{3} a_{1}^{2}-a_{2}}} \ln \left(-\frac{1}{2} \frac{s_{3} A_{3}}{s_{1} A_{1}} \pm \sqrt{\left(\frac{1}{2} \frac{s_{3} A_{3}}{s_{1} A_{1}}\right)^{2}-\frac{s_{2} A_{2}}{s_{1} A_{1}}}\right) .
$$

If

$$
\left(\frac{1}{2} \frac{s_{3} A_{3}}{s_{1} A_{1}}\right)^{2}<\frac{s_{2} A_{2}}{s_{1} A_{1}}
$$

then no extremum exist. If

$$
\left.\begin{array}{c}
\left(\frac{1}{2} \frac{s_{3} A_{3}}{s_{1} A_{1}}\right)^{2}=\frac{s_{2} A_{2}}{s_{1} A_{1}} \\
-\frac{1}{2} \frac{s_{3} A_{3}}{s_{1} A_{1}}>1
\end{array}\right\}
$$

then we have one extremum. If

$$
\left.\begin{array}{c}
\left(\frac{1}{2} \frac{s_{3} A_{3}}{s_{1} A_{1}}\right)^{2}>\frac{s_{2} A_{2}}{s_{1} A_{1}} \\
-\frac{1}{2} \frac{s_{3} A_{3}}{s_{1} A_{1}} \geqslant 1
\end{array}\right\}
$$

then we have two extremums.

$\underline{\text { In the case } 2^{0}}, s_{1}=s_{2}=s_{3}$ the moment of time $\tau$ is determined by the equation

$$
x^{(1)}(\tau)=\left[s_{1} A_{3} \tau^{2}+\left(s_{1} A_{2}+2 A_{3}\right) \tau+\left(s_{1} A_{1}+A_{2}\right)\right] e^{s_{1} \tau}=0
$$

and coefficients $A_{k}$ are

$$
A_{k}=\sum_{i=1}^{k} \frac{x^{(k-i)}(0)(-1)^{i-1} s^{i-1}}{(i-1) !(k-i+1) !}, \quad k=0,1,2, \ldots, n .
$$

For $n=3$ we have

$$
\left.\begin{array}{c}
s=-\frac{1}{3} a_{1} \\
A_{1}=c_{1} \\
A_{2}=\left(c_{2}-s c_{1}\right) \\
\frac{1}{2} c_{3}-s c_{2}-\frac{1}{2} s^{2} c_{1}
\end{array}\right\} .
$$


From the equation (43) we have

$$
\tau=\frac{-\left(s A_{2}+2 A_{3}\right) \pm \sqrt{\left(s A_{2}+2 A_{3}\right)^{2}-4 s A_{3}\left(s A_{1}+A_{2}\right)}}{2 s A_{3}} .
$$

Similarly to the case $1^{0}$ one or two extremums may exist or no extremum exists.

In the case $3^{0}$ :

$$
\left.\begin{array}{c}
s_{3}=\alpha \\
s_{1}=\alpha+j \omega \\
s_{2}=\alpha-j \omega
\end{array}\right\} .
$$

where

$$
\left.\begin{array}{c}
\alpha=-\frac{1}{3} a_{1} \\
\omega=\sqrt{a_{2}-\frac{1}{3} a_{1}^{2}}
\end{array}\right\} .
$$

The equation (34) takes a form

$$
\alpha A_{3} e^{\alpha \tau}+\left[A_{1} \cos \omega \tau+A_{2} \sin \omega \tau\right] e^{\alpha \tau}=0
$$

or after division by $e^{\alpha \tau}$ we obtain

$$
\alpha A_{3}+\left[A_{1} \cos \omega \tau+A_{2} \sin \omega \tau\right]=0 .
$$

From this equation we conclude that there may be infinitely many extremums. In order to obtain the explicit relations of time $\tau$ with the initial conditions $c_{i}(i=1,2,3)$ and roots $s_{1}, s_{2}, s_{3}$ we take into account the relations between coefficients $A_{1}, A_{2}, A_{3}$ with the initial conditions and the roots [5],[6].

In the paper [3] it was proved that the necessary and sufficient condition for the positive and aperiodic solutions of equation (21) is

$1^{0}$

$$
c_{2}=0, c_{1}>0
$$

$2^{0}$ the roots $s_{1}, s_{2}, s_{3}$ are real and negative,

$3^{0}$

$$
\left.\begin{array}{rl}
\frac{c_{3}}{c_{1}}=-s_{1} s_{2} \\
\text { or } \\
\frac{c_{3}}{c_{1}}=-s_{1} s_{3} \\
\text { or } \\
\frac{c_{3}}{c_{1}}=-s_{2} s_{3} .
\end{array}\right\} .
$$


The solution of equation (21) has only one extremum at the moment $\tau=0$, because $c_{2}=\left.\frac{d x}{d t}\right|_{t=0}=0$ by the assumption.

In Fig. 2,3,4 there are presented solutions of (5) for the different $\frac{c_{3}}{c_{1}}$ and $s_{1}, s_{2}, s_{3}$ which fulfill the conditions (50) and (51).

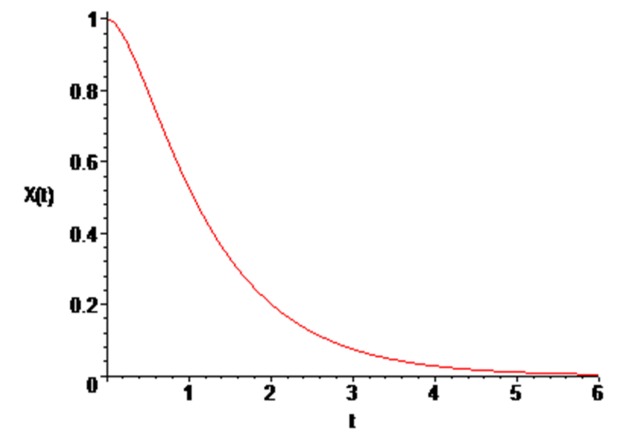

Figure 2: Transient of the error for: $s_{1}=-1, s_{2}=-3, s_{3}=-2, c_{2}=0, c_{3} / c_{1}=-3$.

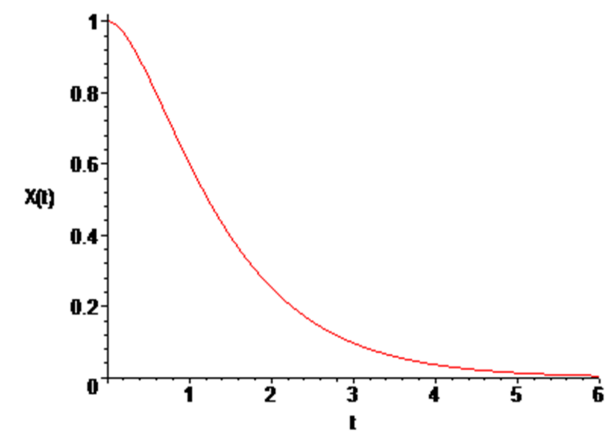

Figure 3: Transient of the error for: $s_{1}=-1, s_{2}=-3, s_{3}=-2, c_{2}=0, c_{3} / c_{1}=-2$.

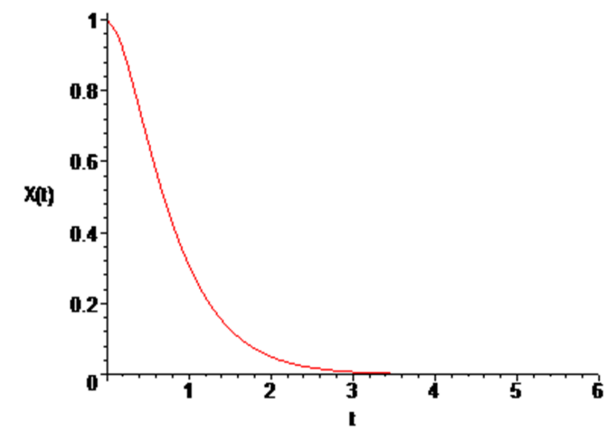

Figure 4: Transient of the error for: $s_{1}=-1, s_{2}=-3, s_{3}=-2, c_{2}=0, c_{3} / c_{1}=-6$. 
In the considerations that follow we assume that

$$
s_{3}=\frac{s_{1}+s_{2}}{2}
$$

and that

$$
s_{2}<s_{3}<s_{1}<0
$$

The solution of equation (21) is

$$
\begin{aligned}
& x(t)= \\
& \frac{\left[s_{2}\left(s_{2}+s_{1}\right) c_{1}-\left(3 s_{2}+s_{1}\right) c_{2}+2 c_{3}\right] e^{s_{1} t}}{\left(s_{1}-s_{2}\right)^{2}}+\frac{\left[s_{1}\left(s_{1}+s_{2}\right) c_{1}-\left(3 s_{1}+s_{2}\right) c_{2}+2 c_{3}\right] e^{s_{2} t}}{\left(s_{1}-s_{2}\right)^{2}}+ \\
& +\frac{\left[-4 s_{1} s_{2} c_{1}+4\left(s_{1}+s_{2}\right) c_{2}-4 c_{3}\right] e^{\left(\frac{s_{1}+s_{2}}{2} t\right)}}{\left(s_{1}-s_{2}\right)^{2}}
\end{aligned}
$$

The derivative of $x(t)$ is

$$
\begin{gathered}
\frac{d x(t)}{d t}=\frac{\left[s_{1} s_{2}\left(s_{2}+s_{1}\right) c_{1}-s_{1}\left(3 s_{2}+s_{1}\right) c_{2}+2 s_{1} c_{3}\right] e^{s_{1} t}}{\left(s_{1}-s_{2}\right)^{2}} \\
+\frac{\left[s_{1} s_{2}\left(s_{1}+s_{2}\right) c_{1}-s_{2}\left(3 s_{1}+s_{2}\right) c_{2}+2 s_{2} c_{3}\right] e^{s_{2} t}}{\left(s_{1}-s_{2}\right)^{2}} \\
+\frac{\left[-2 s_{1} s_{2}\left(s_{1}+s_{2}\right) c_{1}+2\left(s_{1}+s_{2}\right)^{2} c_{2}-2\left(s_{1}+s_{2}\right) c_{3}\right] e^{\left(\frac{s_{1}+s_{2}}{2}\right) t}}{\left(s_{1}-s_{2}\right)^{2}} .
\end{gathered}
$$

Using the necessary condition for extremum $\left.\frac{d x(t)}{d t}\right|_{t=\tau}=0$ we obtain from (55)

$$
\begin{aligned}
& \tau_{1}=\frac{-2}{s_{1}-s_{2}} \ln \left(\frac{\left[-s_{1} s_{2}\left(s_{1}+s_{2}\right) c_{1}+\left(s_{1}+s_{2}\right)^{2} c_{2}-\left(s_{1}+s_{2}\right) c_{3}\right]+\sqrt{A}}{s_{2}\left[-s_{1}\left(s_{1}+s_{2}\right) c_{1}+\left(3 s_{1}+s_{2}\right) c_{2}-2 c_{3}\right]}\right), \\
& \tau_{2}=\frac{-2}{s_{1}-s_{2}} \ln \left(\frac{\left[-s_{1} s_{2}\left(s_{1}+s_{2}\right) c_{1}+\left(s_{1}+s_{2}\right)^{2} c_{2}-\left(s_{1}+s_{2}\right) c_{3}\right]-\sqrt{A}}{s_{2}\left[-s_{1}\left(s_{1}+s_{2}\right) c_{1}+\left(3 s_{1}+s_{2}\right) c_{2}-2 c_{3}\right]}\right),
\end{aligned}
$$

where

$$
A=-\left(s_{1}-s_{2}\right)^{2}\left(-c_{2}^{2} s_{2}^{2}+s_{2}^{2} s_{1} c_{1} c_{2}+2 s_{2} c_{2} c_{3}-3 c_{2}^{2} s_{1} s_{2}+s_{2} s_{1}^{2} c_{1} c_{2}+2 c_{3} s_{1} c_{2}-s_{1}^{2} c_{2}^{2}-c_{3}^{2}\right) .
$$

In Fig. 5 the plot of the solution $x(t)$ is presented for $c_{1}=1, c_{2}=1, c_{3}=3$ and the roots $s_{1}=-1, s_{2}=-3, s_{3}=-2$.

$$
x(t)=7 e^{-t}+4 e^{-3 t}-10 e^{-2 t}
$$

The extremum is only one at the moment of time $\tau=0.69314718$. 


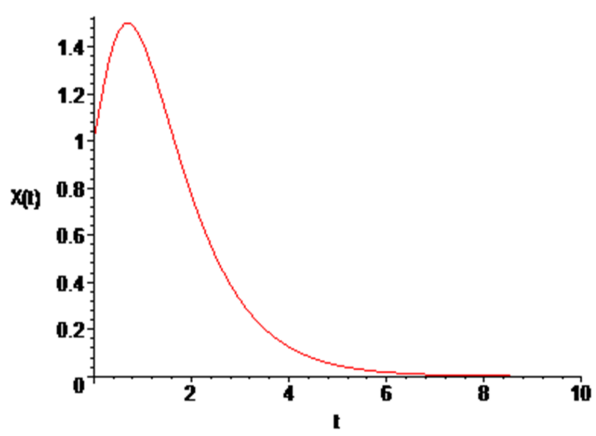

Figure 5: Transient of the error.

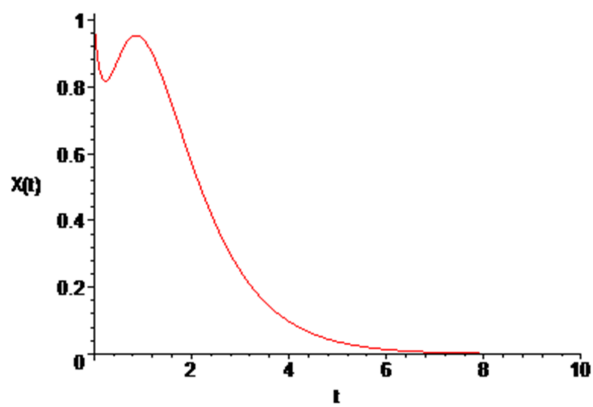

Figure 6: Transient of the error.

In the next example, where $c_{1}=1, c_{2}=-2, c_{3}=15$ we have two extremums at times $\tau_{1}=0.2352329$ and $\tau_{2}=0.863379$. The solution $x(t)$ is presented in Fig. 6 .

$$
x(t)=\frac{11}{2} e^{-t}+\frac{11}{2} e^{-3 t}-10 e^{-2 t} .
$$

In the particular case when $c_{2}=0$ the general formulae (56) and (57) are simpler

$$
\begin{gathered}
\tau_{1}=\frac{-2}{s_{1}-s_{2}} \ln \left(\frac{s_{1} s_{2}^{2} c_{1}+c_{3} s_{2}+s_{1}^{2} s_{2} c_{1}+c_{3} s_{1}+\sqrt{\left(s_{1}-s_{2}\right)^{2} c_{3}^{2}}}{s_{2}\left(s_{1}^{2} c_{1}+s_{1} s_{2} c_{1}+2 c_{3}\right)}\right) \\
=\frac{-2}{s_{1}-s_{2}} \ln \left(\frac{s_{1}\left(s_{2}^{2} c_{1}+s_{1} s_{2} c_{1}+2 c_{3}\right)}{s_{2}\left(s_{1}^{2} c_{1}+s_{1} s_{2} c_{1}+2 c_{3}\right)}\right) \\
\tau_{2}=\frac{-2}{s_{1}-s_{2}} \ln \left(\frac{s_{1} s_{2}^{2} c_{1}+c_{3} s_{2}+s_{1}^{2} s_{2} c_{1}+c_{3} s_{1}-\sqrt{\left(s_{1}-s_{2}\right)^{2} c_{3}^{2}}}{s_{2}\left(s_{1}^{2} c_{1}+s_{1} s_{2} c_{1}+2 c_{3}\right)}\right) \\
=\frac{-2}{s_{1}-s_{2}} \ln \left(\frac{s_{2}\left(s_{1}^{2} c_{1}+s_{1} s_{2} c_{1}+2 c_{3}\right)}{s_{2}\left(s_{1}^{2} c_{1}+s_{1} s_{2} c_{1}+2 c_{3}\right)}\right)=0 .
\end{gathered}
$$


The condition $c_{2}=0$ gives minimal transient error (see [1]). In this particular case when $c_{2}=0$ and $c_{1}=1, c_{3}=3$ extremum is at the moment $\tau_{1}=0$, because $c_{2}=0$, and at the moment of time $\tau_{2}=0.510825$ (Fig.7) and

$$
x(t)=\frac{9}{2} e^{-t}+\frac{5}{2} e^{-3 t}-6 e^{-2 t} .
$$

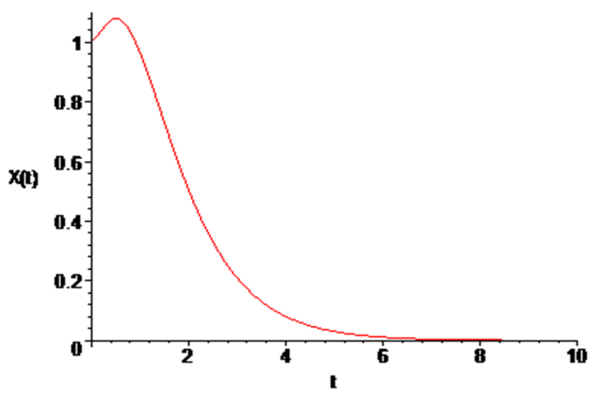

Figure 7: Transient of the error.

The case $2^{0}$

$$
s_{1}=s_{2}=s_{3}=-\frac{1}{3} a_{1}
$$

The solution is

$$
x(t)=\left[\left(\frac{1}{2} c_{3}+\frac{1}{2} s_{1}^{2} c_{1}-s_{1} c_{2}\right) t^{2}+\left(c_{2}-s_{1} c_{1}\right) t+c_{1}\right] e^{s_{1} t} .
$$

The derivative

$$
\frac{d x(t)}{d t}=\left[\left(\frac{1}{2} s_{1} c_{3}+\frac{1}{2} s_{1}^{3} c_{1}-s_{1}^{2} c_{2}\right) t^{2}+\left(c_{3}-s_{1} c_{2}\right) t+c_{2}\right] e^{s_{1} t} .
$$

From the condition that $\frac{d x(t)}{d t}=0$ we obtain

$$
\begin{gathered}
\tau_{1}=\frac{-c_{3}+s_{1} c_{2}+\sqrt{c_{3}^{2}-4 c_{3} c_{2} s_{1}+5 s_{1}^{2} c_{2}^{2}-2 c_{2} c_{1} s_{1}^{3}}}{s_{1} c_{3}+s_{1}^{3} c_{1}-2 s_{1}^{2} c_{2}}, \\
\tau_{2}=\frac{-c_{3}+s_{1} c_{2}-\sqrt{c_{3}^{2}-4 c_{3} c_{2} s_{1}+5 s_{1}^{2} c_{2}^{2}-2 c_{2} c_{1} s_{1}^{3}}}{s_{1} c_{3}+s_{1}^{3} c_{1}-2 s_{1}^{2} c_{2}} .
\end{gathered}
$$

For numerical example according to $s_{1}=-1, c_{1}=1, c_{2}=1, c_{3}=2$ the solution of $x(t)$ is presented in Fig. 8.

$$
x(t)=\left(\frac{5}{2} t^{2}+2 t+1\right) e^{-t}
$$




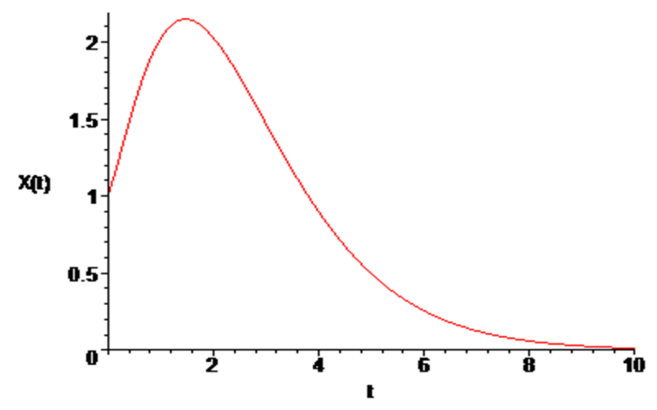

Figure 8: Transient of the error.

and from (64) we have $\tau=1.471779$.

In the case when $c_{2}=0$ the formulae (63) and (64) are

$$
\begin{gathered}
\tau_{1}=\frac{1}{2}\left(\frac{-2 c_{3}+2 \sqrt{c_{3}^{2}}}{s_{1} c_{3}+s_{1}^{3} c_{1}}\right)=0, \\
\tau_{2}=\frac{-2 c_{3}}{s_{1}\left(c_{3}+s_{1}^{2} c_{1}\right)} .
\end{gathered}
$$

The case $3^{0}$

$$
s_{3}=\alpha, s_{1}=\alpha+j \omega, s_{2}=\alpha-j \omega, c_{1} \neq 0, c_{2}=0, c_{3} \neq 0 .
$$

The solution is

$$
x(t)=-\frac{e^{\alpha t}\left[-c_{1} \alpha^{2}-c_{1} \omega^{2}-c_{3}+\cos (t \omega) c_{1} \alpha^{2}+c_{3} \cos (t \omega)+\alpha c_{1} \omega \sin (t \omega)\right.}{\omega^{2}} .
$$

The derivative

$$
\begin{aligned}
& \frac{d x(t)}{d t}= \\
& -\frac{e^{\alpha t}\left[-c_{1} \alpha^{3}-\alpha c_{1} \omega^{2}-\alpha c_{3}+\alpha^{3} c_{1} \cos (t \omega)+\alpha c_{1} \omega^{2} \cos (t \omega)+\alpha c_{3} \cos (t \omega)-c_{3} \omega \sin (t \omega)\right]}{\omega^{2}}
\end{aligned}
$$

or in a more convenient form

$$
\frac{d x(t)}{d t}=\left[\frac{c_{3} \sin (t \omega)}{\omega}+\frac{\left(c_{1} \alpha^{3}+\alpha c_{1} \omega^{2}+\alpha c_{3}\right)[1-\cos (t \omega)]}{\omega^{2}}\right] e^{\alpha t} .
$$

From the necessary condition $\frac{d x(t)}{d t}=0$ using (70) we obtain

$$
\tau_{1}=2 \frac{k \pi}{\omega}, k=0,1, \ldots
$$




$$
\tau_{2}=\frac{-2}{\omega} \operatorname{arctg}\left(\frac{c_{3} \omega}{\alpha\left(c_{1} \alpha^{2}+c_{1} \omega^{2}+c_{3}\right)}\right)+\frac{2 k \pi}{\omega}, \quad k=0,1, \ldots
$$

where for $\alpha=-\frac{1}{3} a_{1}, \omega=\sqrt{a_{2}-\frac{1}{3} a_{1}^{2}}$ we obtain finally from (72)

$$
\tau_{2}=6 \frac{-\operatorname{arctg}\left(9 \frac{c_{3} \sqrt{\left(9 a_{2}-3 a_{1}^{2}\right.}}{a_{1}\left(-9 c_{3}+2 c_{1} a_{1}^{2}-9 c_{1} a_{2}\right)}\right)+k \pi}{\sqrt{9 a_{2}-3 a_{1}^{2}}} .
$$

A numerical example for $\alpha=-1, \omega=2, c_{1}=1, c_{2}=0, c_{3}=-3$ is presented in Fig. 9 and the solution is

$$
x(t)=\frac{1}{2}[1+\cos (2 t)+\sin (2 t)] e^{-t}
$$

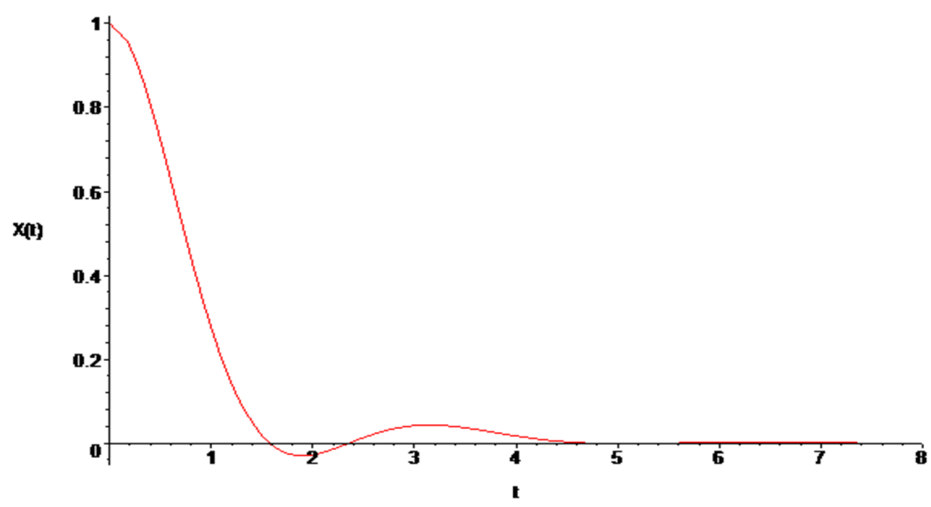

Figure 9: Transient of the error.

Extremums from (71) and (72) are as follows: $\tau_{1}=0, \tau_{2}=1.89254688, \tau_{3}=$ $3.14159265, \tau_{4}=5.03413953, \tau_{5}=6.2831853$.

For $c_{2}=0$, the example presented in the paper [2] can be described by the following relations:

Transform of the error

$$
E(s)=\frac{1+2 \xi T_{3} s+T_{3}^{2} s^{2}}{K+\left(1+K T_{2}\right) s+2 \xi T_{3} s^{2}+T_{3}^{2} s^{3}} .
$$

The initial conditions are

$$
c_{1}=1, \quad c_{2}=0, \quad c_{3}=-\frac{K T_{2}}{T_{3}^{2}} .
$$

For numerical values: $\xi=0.75, T_{3}=0.1, K=10, T_{2}=0.15$ we obtain

$$
X(s)=\frac{1+0.15 s+0.01 s^{2}}{10+2.5 s+0.15 s^{2}+0.01 s^{3}} .
$$


Solution

$$
\begin{aligned}
& x(t)=[0.2857142857+0.7142857143 \cos (13.22875656 t)+ \\
&+0.3779644731 \sin (13.22875656 t)] e^{-5 t}
\end{aligned}
$$

is presented in Fig. 10.

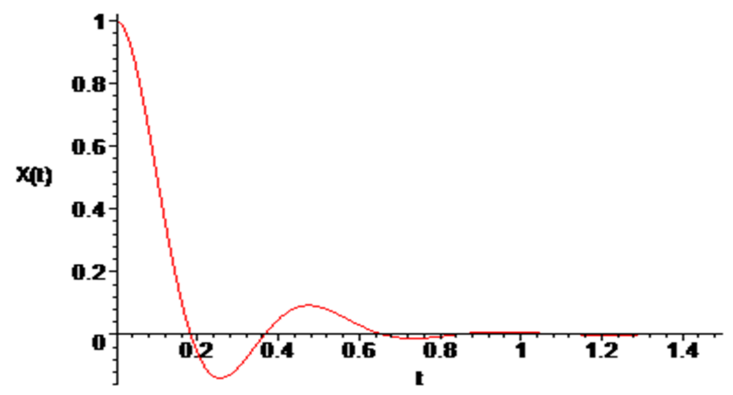

Figure 10: Transient of the error.

From (71) and (72) the extremums of $x(t)$ are at the moments of time $\tau_{1}=0, \tau_{2}=$ $0.2564298695, \tau_{3}=0.4749641647, \tau_{4}=0.7313940343, \tau_{5}=0.9499283295$ and so on. The values of the extremum are: $x\left(\tau_{1}\right)=1, x\left(\tau_{2}\right)=-0.13872, x\left(\tau_{3}\right)=0.093031, x\left(\tau_{4}\right)=$ $-0.012905, x\left(\tau_{5}\right)=0.0086548$.

\section{Conclusions}

It was shown that also in the difficult case when $c_{2}=0$ it is possible to obtain solution of the problem. In the article [1] it was proved that the condition $c_{2}=0$ gives minimal dynamic error for the $n$th order equation $(n=2,3, \ldots)$. For that reason this particular case is very important. The 3-rd order equation was analyzed and for the different kinds of roots the analytical formulae of the extremum of dynamic error $x(\tau)$ and time $\tau$ has been obtained. In the figures there are shown the transients of $x(t)$. The practical example, which was considered in [2], is also solved for the initial conditions $c_{1}=1$, $c_{2}=0$. The roots of the characteristic equation may be shifted in the desired location using the well known methods of the poles and zeros locations (see [7]).

\section{References}

[1] H. GóreCKI and A. Turowicz: Optimum transient problem in linear automatic control system. Proc. of the 1st IFAC Congress, Moscow, pp.59-61, Published by Butterworths London, (1960). 
[2] H. GóReCKI and M. ZACZYK: Decomposition method and its application to the extremal problems. Archives of Control Sciences, 26(1) (2016), 49-67.

[3] H. GóRECKI: Analytic solution of transcendental equations. Int. J. Applied Mathematics and Computer Science, 20(4), (2010), 671-677.

[4] H. GóRECKI and M. ZACZYK: Design of systems with extremal dynamic properties. Bulletin of the Polish Academy of Sciences, Technical Sciences, 61(3), (2013), 563-567.

[5] H. GóRECKI: Algebraic condition for decomposition of large-scale linear dynamic systems. Int. J. Applied Mathematics and Computer Science, 19(1), (2009), 107111.

[6] H. GóReCKi AND M. ZACZYK: Design of the oscillatory systems with the extremal dynamic properties. Bulletin of the Polish Academy of Sciences, Technical Sciences, 62(2), (2014), 241-253.

[7] T. KACZOREK: Theory of control. PWN, Warsaw (1977), (in Polish).

[8] H. Kobayashi: Output overshoot and pole-zero configuration. Proc. 12th IFAC Congress, Sydney, 3 (1993), 73-76.

[9] H. Kobayashi and E. Shimemura: Some properties of optimal regulators and their applications. Int. J. Control, 33(4), (1981), 587-599.

[10] W. MitKowsKi: Stability of Dynamical Systems. PWN, Warsaw (1991), (in Polish).

[11] I. KoźNIEWsKa: Recurent Equations. PWN, Warsaw (1972), (in Polish).

[12] M. ZIóŁKo: Modeling of Wave Phenomena. AGH, University Press, Krakow (2000), (in Polish). 